\title{
COHOMOLOGY OF GROUPS IN ARBITRARY CATEGORIES
}

\author{
BODO PAREIGIS ${ }^{1}$
}

1. Introduction. In this paper we give a short sketch of a method of doing cohomology theory of group-like objects in arbitrary categories. The way of approach is closely connected with the usual theory of cohomology of groups and has also been used by D. K. Harrison in [6]. Specifically the equivalence of the homogeneous, inhomogeneous, and normalized theories will be shown. Since we consider arbitrary categories we must give all definitions by properties of maps and cannot apply explicit computations with elements. But instead of using the diagrams which contain the maps of those definitions, we consider these maps as elements of the morphism sets and use the algebraic structures of the morphism sets which are induced by the abstractly defined structures of the objects.

In [6] two examples of this theory, Harrison's complex and Amitsur's complex, have already been mentioned. Since the homogeneous and inhomogeneous definitions of Harrison's complex are equivalent we can prove that Harrison's complex is a subcomplex of Amitsur's complex.

This theory may be developed in greater generality using certain functorial properties of the cohomology theory of groups as I. Berstein pointed out to me. I hope that the possibilities of explicit computation as described in this paper, might also be of some interest.

2. Notation. Let $\mathfrak{C}$ be a category with finite direct products. This means that for a finite collection of objects $B_{1}, \cdots, B_{n}$ in $\mathfrak{e}$ there exists an object $\prod B_{i}=B_{1} \times \cdots \times B_{n}$ in $\mathcal{e}$ and morphisms $p_{\boldsymbol{i}}: \prod B_{\boldsymbol{i}}$ $\rightarrow B_{i}$, so called projections, such that for any object $A$ in $\mathcal{C}$ and any system of morphisms $b_{i}: A \rightarrow B_{i}, i=1, \cdots, n$ there exists a unique morphism $b: A \rightarrow \prod B_{i}$ with $p_{i} b=b_{i}$. We write $\left(b_{1}, \cdots, b_{n}\right)$ instead of $b$. It is easy to see that $\left(p_{1}, \cdots, p_{n}\right)=\mathrm{id}$, the identity on $\prod B_{i}$. The direct product of $n$ copies of $B$ will be written as $B^{n}$. Furthermore we denote by $\operatorname{Mor}(A, B)$ the set of morphisms from $A$ to $B$. For any finite set of morphisms $b_{i} \in \operatorname{Mor}\left(A_{i}, B_{i}\right), i=1, \cdots, n$ we denote by $\left(b_{1} \times \cdots \times b_{n}\right)$ the morphism $\left(b_{1} p_{1}, \cdots, b_{n} p_{n}\right)$ $\in \operatorname{Mor}\left(\prod A_{i}, \prod B_{i}\right)$, where $p_{j} \in \operatorname{Mor}\left(\prod A_{i}, A_{j}\right)$. For the composi-

Received by the editors May 11, 1963.

1 The author was supported by NATO-Research-Fellowship 4-s-nato $2 / 3$ gf. 
tion of these morphisms we have the following rules [3, Propositions 3.5, 3.6, 3.7]: let $b \in \operatorname{Mor}(A, B), c_{i} \in \operatorname{Mor}\left(B, C_{i}\right), d_{i} \in \operatorname{Mor}\left(C_{i}, D_{i}\right)$, and $e_{i} \in \operatorname{Mor}\left(D_{i}, E_{i}\right)$, then

(a) $\left(c_{i}, \cdots, c_{n}\right) b=\left(c_{1} b, \cdots, c_{n} b\right)$,

$$
\begin{aligned}
& \text { (b) }\left(d_{1} \times \cdots \times d_{n}\right)\left(c_{1}, \cdots, c_{n}\right)=\left(d_{1} c_{1}, \cdots, d_{n} c_{n}\right) \text {, } \\
& \text { (c) }\left(e_{1} \times \cdots \times e_{n}\right)\left(d_{1} \times \cdots \times d_{n}\right)=\left(e_{1} d_{1} \times \cdots \times e_{n} d_{n}\right) \text {. }
\end{aligned}
$$

Since we do not assume that the category $\mathfrak{C}$ is a category with zeros in the sense of [3] we have to use another axiom for the neutral element for group-like structures.

The complete set of axioms which we shall use is:

(I) There exists a morphism $\mu: X \times X \rightarrow X$. $\mu$ is called a multiplication on $X$.

(II) There exists an element $0 \in \operatorname{Mor}(X, X)$ such that

(a) $0 f=0 g$ for all objects $A$ and all $f, g \in \operatorname{Mor}(A, X)$,

(b) $\mu(0$, id $)=$ id $\in \operatorname{Mor}(X, X)$,

(c) $\mu(\mathrm{id}, 0)=\mathrm{id} \in \operatorname{Mor}(X, X)$.

(III) $\mu(\mu \times \mathrm{id})=\mu(\mathrm{id} \times \mu) \in \operatorname{Mor}\left(X^{3}, X\right)$.

(IV) There exists a morphism $s \in \operatorname{Mor}(X, X)$ such that $\mu($ id, $s)$ $=\mu(s, \mathrm{id})=0 \in \operatorname{Mor}(X, X)$.

(V) Let id $=\left(p_{1}, p_{2}\right)$ and $\tau=\left(p_{2}, p_{1}\right) \in \operatorname{Mor}\left(X^{2}, X^{2}\right)$, then $\mu=\mu \tau$.

If (I) and (III) hold, then $X$ is called a semigroup, if (I), (II), (III), and (IV) hold, then $X$ is called a group and if (I), (II), (III), (IV) and $(\mathrm{V})$ hold, then $X$ is called a commutative group. We write $(X, \mu)$ to indicate that $\mu$ is the multiplication on $X$ under consideration.

One easily proves that $0 \in \operatorname{Mor}(A, A)$ is unique. We call 0 the neutral element of the multiplication $\mu$. If the necessary axioms hold for the multiplication $\mu$ in $X$, we shall use the following notation with $x_{i} \in \operatorname{Mor}(A, X)$

$$
\begin{aligned}
& \text { (a) } \mu\left(x_{1}, x_{2}\right)=x_{1} \cdot x_{2} \quad\left(=x_{1}+x_{2}\right) \text {, } \\
& \text { (b) } 0 x_{i}=1 \quad(=0) \text {, } \\
& \text { (c) } \quad s x_{i}=x_{i}^{-1} \quad\left(=-x_{i}\right) \text {. }
\end{aligned}
$$

The notation in parentheses will be used, if the multiplication is commutative, i.e., if axiom (V) holds.

Let now $(X, \mu)$ be a semigroup and $(Y, \nu)$ a commutative group and $\lambda \in \operatorname{Mor}(X \times Y, Y)$. Then we shall write for all $A$ and all morphisms $x \in \operatorname{Mor}(A, X), y \in \operatorname{Mor}(A, Y)$

$$
\lambda(x, y)=x \cdot y \text {. }
$$


This notation should not interfere with the notation (2.2.a).

We call $Y$ an $X$-module, if $Y$ is a commutative group and $X$ operates on $Y$ as in (2.3) and if for all $x, x_{1}, x_{2} \in \operatorname{Mor}(A, X) ; y, y_{1}, y_{2}$ $\in \operatorname{Mor}(A, Y)$

(a) $x \cdot\left(y_{1}+y_{2}\right)=x \cdot y_{1}+x \cdot y_{2}$,

(b) $\left(x_{1} \cdot x_{2}\right) \cdot y=x_{1} \cdot\left(x_{2} \cdot y\right)$

and if $\operatorname{Mor}(X, Y) \neq \varnothing$.

We shall call the $X$-module structure on $Y$ trivial if $\lambda(x, y)=y$ for all $A$ and all $x \in \operatorname{Mor}(A, X)$, ultratrivial if $\lambda(x, y)=0$ for all $A$ and all $x \in \operatorname{Mor}(A, X)$. If $(X, \mu)$ has a neutral element 1 and if $\lambda(1, y)=y$, then we call $Y$ a unitary $X$-module. Certainly a trivial $X$-module structure on $Y$ implies that $Y$ is a unitary $X$-module. We remark furthermore that with the above definitions $\operatorname{Mor}(A, Y)$ is a group if $Y$ is a group [3, Theorem 4.10].

All these definitions are already well known [3] but by using the notation (2.2) and (2.3) computation will become easier and we can easily refer to computations made in the classical case. Thus axioms (2.4) are already given in this notation and stand for certain commutative diagrams.

3. Cohomology of groups. In the notation given in the preceding paragraph it is now easy to generalize the definition of the cohomology of a semigroup $(X, \mu)$ with coefficients in an $X$-module $(Y, \nu)$. We define the differentiation $\partial^{n}: \operatorname{Mor}\left(X^{n}, Y\right) \rightarrow \operatorname{Mor}\left(X^{n+1}, Y\right)$ by

$$
\begin{aligned}
\partial^{n}(f)= & \partial^{n}(f)\left(p_{1}, \cdots, p_{n+1}\right) \\
= & p_{1} \cdot\left(f\left(p_{2}, \cdots, p_{n+1}\right)\right) \\
& +\sum_{i=1}^{n}(-1)^{i} f\left(p_{1}, \cdots, p_{i} \cdot p_{i+1}, \cdots, p_{n+1}\right) \\
& +(-1)^{n+1} f\left(p_{1}, \cdots, p_{n}\right),
\end{aligned}
$$

where $p_{i} \in \operatorname{Mor}\left(X^{n+1}, X\right), f \in \operatorname{Mor}\left(X^{n}, Y\right)$ and $(-1)^{i f}=s^{i} f$. As in ordinary cohomology theory [4] one checks $\partial^{n+1} \partial^{n}(f)=0$ and that $\partial^{n}$ is a homomorphism. Furthermore we define a set which we denote by $\operatorname{Mor}\left(X^{0}, Y\right)=\operatorname{Mor}(Y, Y) 0$, where $0 \in \operatorname{Mor}(X, Y)$. It is easy to see that $\operatorname{Mor}\left(X^{0}, Y\right)$ is a commutative group under the induced multiplication of $Y$. We define $\partial^{0}: \operatorname{Mor}\left(X^{0}, Y\right) \rightarrow \operatorname{Mor}(X, Y)$ by

$$
\partial^{0}(f 0)(p)=p \cdot(f 0)-(f 0) \in \operatorname{Mor}(X, Y),
$$

where $(p)=\mathrm{id} \in \operatorname{Mor}(X, X) . \partial^{0}$ is a homomorphism too and we get $\partial^{1} \partial^{0}(f 0)=0$. 
If the category $\mathcal{C}$ has a final object $F[5$, p. 332], i.e., if there exists an object $F$ such that $\operatorname{Mor}(A, F)$ contains exactly one element for all $A$ in $\mathfrak{C}$, and if $\operatorname{Mor}(F, Y)$ is nonempty, then $0 \in \operatorname{Mor}(Y, Y)$ admits the factorization

$$
Y \rightarrow F \stackrel{0}{\rightarrow} Y
$$

and $\operatorname{Mor}\left(X^{0}, Y\right) \cong \operatorname{Mor}(F, Y)$ by a natural isomorphism, which explains the definition of $\operatorname{Mor}\left(X^{0}, Y\right)$. In the examples in $\$ 4$ we always shall have categories with a final object.

We thus have constructed a complex of abelian groups:

$$
0 \stackrel{\partial^{-1}}{\rightarrow} \operatorname{Mor}\left(X^{0}, Y\right) \stackrel{\partial^{0}}{\rightarrow} \operatorname{Mor}(X, Y) \stackrel{\partial^{1}}{\rightarrow} \operatorname{Mor}\left(X^{2}, Y\right) \cdots
$$

We define the inhomogeneous cohomology groups of $X$ with coeffcients in $Y$ by

$$
H^{n}(X, Y)=\operatorname{Ker}^{n} / \operatorname{Im} \partial^{n-1}, \quad n \geqq 0 .
$$

We also can define the homogeneous cohomology groups. For this purpose we consider the set of morphisms $f \in \operatorname{Mor}\left(X^{n+1}, Y\right)$ with the property

$$
p \cdot\left(f\left(p_{0}, \cdots, p_{n}\right)\right)=f\left(p \cdot p_{0}, \cdots, p \cdot p_{n}\right)
$$

and denote this set by $\operatorname{Mor}_{X}\left(X^{n+1}, Y\right)$. One can easily prove that this definition implies

$$
x \cdot\left(f\left(x_{0}, \cdots, x_{n}\right)\right)=f\left(x \cdot x_{0}, \cdots, x \cdot x_{n}\right)
$$

for $x, x_{i} \in \operatorname{Mor}(A, X)$. Obviously $\operatorname{Mor}_{X}\left(X^{n+1}, Y\right)$ is still a commutative group. We define homomorphisms

by

$$
\delta^{n}: \operatorname{Mor}_{X}\left(X^{n}, Y\right) \rightarrow \operatorname{Mor}_{X}\left(X^{n+1}, Y\right), \quad n \geqq 1
$$

$$
\delta^{n}(f)\left(p_{0}, \cdots, p_{n}\right)=\sum_{i=0}^{n}(-1)^{i} f\left(p_{0}, \cdots, \hat{p}_{i}, \cdots, p_{n}\right),
$$

where - means that the projection under this sign is to be omitted. Here again one easily checks that $\delta^{n+1} \delta^{n}(f)=0$. Thus one obtains the complex

$$
0 \stackrel{\delta^{0}}{\rightarrow} \operatorname{Mor}_{X}(X, Y) \stackrel{\delta^{1}}{\rightarrow} \operatorname{Mor}_{X}\left(X^{2}, Y\right) \stackrel{\delta^{2}}{\rightarrow} \cdots
$$

and defines homogeneous cohomology groups by

$$
\bar{H}^{n}(X, Y)=\operatorname{Ker} \delta^{n+1} / \operatorname{Im} \delta^{n}, \quad n \geqq 0 .
$$

TheOREM 3.1. Let $(X, \mu)$ be a group and $(Y, \nu)$ a unitary $X$-module 
and let $\operatorname{Mor}(Y, X) \neq \varnothing$. Then

$$
\bar{H}^{n}(X, Y) \cong H^{n}(X, Y), \quad n \geqq 0 .
$$

Proof. This proof is exactly the same as in the ordinary cohomology of groups [4]; one proves that $\operatorname{Mor}_{X}\left(X^{n+1}, Y\right) \cong \operatorname{Mor}\left(X^{n}, Y\right)$ and that the differentiation operators $\delta^{n}$ and $\partial^{n}$ commute with these isomorphisms.

If now $(X, \mu)$ is a semigroup with neutral element and $(Y, \nu)$ is an $X$-module then we consider those elements $f \in \operatorname{Mor}\left(X^{n}, Y\right)$ with $f\left(x_{1}, \cdots, x_{n}\right)=0$ if one of the $x_{i} \in \operatorname{Mor}(A, X)$ is the neutral element. This subset forms a group $\operatorname{Mor}^{N}\left(X^{n}, Y\right)$ of normalized cochains for $n \geqq 1$. For $n=0$ we define $\operatorname{Mor}^{N}\left(X^{0}, Y\right)=\operatorname{Mor}\left(X^{0}, Y\right)$. If we denote the differentiation induced by $\partial^{n}$ on these subgroups also by $\partial^{n}$ we get the complex

$$
0 \stackrel{\partial^{-1}}{\longrightarrow} \operatorname{Mor}^{N}\left(X^{0}, Y\right) \stackrel{\partial^{0}}{\rightarrow} \operatorname{Mor}^{N}(X, Y) \stackrel{\partial^{1}}{\rightarrow} \operatorname{Mor}^{N}\left(X^{2}, Y\right) \cdots
$$

and the cohomology groups

$$
\hat{H}^{n}(X, Y)=\operatorname{Ker} \partial^{n} / \operatorname{Im} \partial^{n-1}, \quad n \geqq 0 .
$$

As in the classical case [4] one proves

Proposition 3.2. Let $(X, \mu)$ be a semigroup with neutral element and $(Y, \nu)$ a unitary $X$-module. Then

$$
\hat{H}^{n}(X, Y) \cong H^{n}(X, Y), \quad n \geqq 0 .
$$

4. Examples. Let us now consider two examples of this theory. In the first example let $\mathfrak{C}$ be the category of sets and set maps; then we get the ordinary cohomology of groups.

Another example is the following, due to Harrison [6]: For a commutative ring $K$ with identity consider the category $Q$ of commutative $K$-algebras with identity and $K$-algebra homomorphisms which preserve the identity. Let $\mathbb{C}=\mathfrak{Q}^{0}$ be the dual of this category. Since a has finite inverse products, namely the tensor products of $K$ algebras, $\mathfrak{e}$ has finite direct products. Since $a$ has an initial object $K$ (in the sense of [5]), i.e., every set of $K$-algebra homomorphisms from $K$ to any $K$-algebra $A$ consists of exactly one element, $\mathfrak{e}$ has a final object $K^{0}$.

Let now $A$ be any arbitrary $K$-algebra in $a,\langle z\rangle=Z$ the infinite cyclic group with generator $z$, and $G$ any commutative multiplicative group. We denote by $K(Z)$ and $K(G)$ the group rings of $Z$ and $G$ over $K$. Then the following definitions make $A^{0}$ into a semigroup, $K(Z)^{0}$ and $K(G)^{0}$ into groups, and induce an ultratrivial $A^{0}$-module structure and a trivial $K(G)^{0}$-module structure on $K(Z)^{0}$ : 


$$
\begin{aligned}
& \mu_{1}: \quad A \rightarrow A \otimes A \quad \mu_{1}(a)=1 \otimes a \\
& \mu_{2}: K(G) \rightarrow K(G) \otimes K(G) \quad \mu_{2}(g)=g \otimes g \\
& \nu: K(Z) \rightarrow K(Z) \otimes K(Z) \quad \nu(z)=z \otimes z \\
& \lambda_{1}: K(Z) \rightarrow A \otimes K(Z) \quad \lambda_{1}(z)=1 \otimes 1 \\
& \lambda_{2}: K(Z) \rightarrow K(G) \otimes K(Z) \quad \lambda_{2}(z)=1 \otimes z .
\end{aligned}
$$

We notice that all $K$-algebra homomorphisms from $K(Z)$ to $A$ are uniquely determined by the image of the generator $z$ of $Z$ and that the range for the images of $z$ is just the group of units $A^{*}$ of $A$. So we get $\operatorname{Mor}\left(\left(A^{0}\right)^{n}, K(Z)^{0}\right) \cong\left(A^{n}\right)^{*}$.

If we evaluate the inhomogeneous differentiation operators $\partial^{n}$, we get Amitsur's complex ([1] and [7])

$$
\mathfrak{U}(A / K): 1 \rightarrow K^{*} \stackrel{\Delta^{0}}{\rightarrow} A^{*} \stackrel{\Delta^{1}}{\rightarrow}(A \otimes A)^{*} \stackrel{\Delta^{2}}{\rightarrow} \cdots
$$

from the $A^{0}$-module $K(Z)^{0}$. For the $K(G)^{0}$-module $K(Z)^{0}$ we get

$$
\mathfrak{S}(G): 1 \rightarrow K^{*} \stackrel{\nabla^{0}}{\rightarrow} K(G) * \stackrel{\nabla^{1}}{\rightarrow}(K(G) \otimes K(G))^{*} \stackrel{\nabla^{2}}{\rightarrow} \ldots
$$

which is Harrison's complex [6] in case $G=\boldsymbol{Q} / \boldsymbol{Z}$ and $K$ is a field, where $\boldsymbol{Q}$ are the rational numbers and $\boldsymbol{Z}$ are the integers.

This example has already been given by Harrison in [6], but the definitions are repeated for the convenience of the reader.

By Theorem 3.1 we can also apply the homogeneous cohomology theory for the complex (4.2). By evaluating the maps $\delta^{n}$ we easily verify that this complex may be regarded as a subcomplex of Amitsur's complex $\mathfrak{A}(K(G) / K)$. Indeed the subgroups which form the complex (4.2) in the homogeneous representation consist of all elements of $\left(K(G)^{n}\right)^{*}$ of the form

$$
\sum_{i=1}^{m} k_{i} g_{1, i} \otimes \cdots \otimes g_{n, i}
$$

such that $\prod_{j=1}^{n} g_{j, i}=1$ for all $i$. In case $G=\boldsymbol{Q} / \boldsymbol{Z}$ and $q_{j, i} \in \boldsymbol{Q} / \boldsymbol{Z}$, we get that $\mathfrak{S}(\boldsymbol{Q} / \boldsymbol{Z})$ is a subcomplex of $\mathfrak{A}(K(\boldsymbol{Q} / \boldsymbol{Z}) / K)$ and that the last conditions read:

$$
\sum_{i=1}^{m} k_{i} q_{1, i} \otimes \cdots \otimes q_{n, i} \in\left(K(\boldsymbol{Q} / Z)^{n}\right)^{*}
$$

such that $\sum_{j=1}^{n} q_{j, i}=0$ for all $i$.

Thus we get a factor complex $\mathfrak{U}$ of $\mathfrak{A}=\mathfrak{U}(K(\mathbb{Q} / \boldsymbol{Z}) / K)$ over $\mathfrak{S}=\mathfrak{S}(\boldsymbol{Q} / \boldsymbol{Z})$ and an exact sequence

$$
1 \rightarrow \mathfrak{E} \rightarrow \mathfrak{A} \rightarrow \mathfrak{U} \rightarrow 1
$$


THEOREM 4.1. $H^{n}(\mathfrak{)}) \cong H^{n-1}(\mathfrak{U}), \quad n \geqq 1$.

Proof. The exact sequence (4.3) gives rise to an exact cohomology sequence

$$
\cdots \rightarrow H^{n}(\mathfrak{H}) \rightarrow H^{n}(\mathfrak{U}) \rightarrow H^{n-1}(\mathfrak{S}) \rightarrow H^{n-1}(\mathfrak{H}) \rightarrow \cdots .
$$

We want to prove $H^{n}(\mathfrak{U})=0$ for all $n$.

For $H^{n}(\mathfrak{U})$ we have $H^{n}(\mathfrak{U})=H^{n}(K(\boldsymbol{Q} / \boldsymbol{Z}) / K)$, and $K(\boldsymbol{Q} / \boldsymbol{Z})$ $\cong \lim _{\rightarrow} K(G)$ for finite cyclic groups $G$ and by $\left[7\right.$, p. 345] $H^{n}(\mathfrak{H})$ $\cong \lim _{\rightarrow} H^{n}(K(G) / K)$.

Now let $G$ be a cyclic group and 1 be the unit element of $G$. We define $\alpha: K \rightarrow K(G)$ by $\alpha(k)=k \cdot 1$ where $k \in K$, and $\beta: K(G) \rightarrow K$ by $\beta\left(\sum k_{i} g^{i}\right)=\sum k_{i}$, where $k_{i} \in K$ and $g$ the generator of $G$. Then as in the proof of $[9$, Lemma 3.1] we have that the chain maps defined by $\alpha$ and $\beta$ induce maps $\alpha^{*}: H^{n}(K / K) \rightarrow H^{n}(K(G) / K)$ and $\beta^{*}$ : $H^{n}(K(G) / K) \rightarrow H^{n}(K / K)$ which are isomorphisms. So $H^{n}(K(G) / K)$ $=0$ for all finite cyclic groups $G$ and thus $H^{n}(K(\boldsymbol{Q} / Z) / K)=0$. By the exact cohomology sequence (4.4) we get the desired result of Theorem 4.1 .

We have studied the homogeneous form of Harrison's complex. If one tries to construct homogeneous cohomology groups for Amitsur's cohomology one will find that the cohomology groups vanish, due to the more general fact that for ultratrivial module structures the complex (3.2) vanishes.

\section{BIBLIOGRAPHY}

1. S. A. Amitsur, Simple algebras and cohomology groups of arbitrary fields, Trans. Amer. Math. Soc. 90 (1959) 73-112.

2. A. J. Berkson, On Amitsur's complex and restricted Lie algebras, Trans. Amer. Math. Soc. 109 (1963), 430-443.

3. B. Eckmann and P. J. Hilton, Group-like structures in general categories. I. Multiplications and comultiplications, Math. Ann. 145 (1962), 227-255.

4. S. Eilenberg and S. MacLane, Cohomology theory in abstract groups. I, Ann. of Math. (2) 48 (1947), 326-341. 448.

5. P. Gabriel, Des catégories abéliennes, Bull. Soc. Math. France 90 (1962), 323-

6. D. K. Harrison, Abelian extensions of arbitrary fields, Trans. Amer. Math. Soc. 106 (1963), 230-235.

7. A. Rosenberg and D. Zelinsky, On Amitsur's complex, Trans. Amer. Math. Soc. 97 (1960), 327-356.

8. - A mitsur's complex for inseparable fields, Osaka Math. J. 14 (1962), 219240.

9. B. Pareigis and A. Rosenberg, Addendum to "Amitsur's complex for purely inseparable fields," Osaka Math. J. (to appear).

Cornell University and

UNIVERSITÄT HEIDELBERG 